\section{Rheumatoide Arthritis und Diabetes Typ 2}

Jin Y et al. Risk of Incident Type 2 Diabetes Mellitus Among Patients With Rheumatoid Arthritis: A Population-Based Cohort Study. Arthritis Care Res (Hoboken) 2020; 72 : 1248-1256. doi: 10.1002/acr.24343

Ob Patienten mit einer rheumatoiden Arthritis (RA) überproportional häufig an einem Diabetes mellitus Typ 2 erkranken, wird in der wissenschaftlichen Literatur kontrovers diskutiert. US-Forscher untersuchten diese Fragestellung nun im Rahmen einer retrospektiven Kohortenstudie. Sie bildeten dabei die Diabetesinzidenz in 4 verschiedenen Vergleichsgruppen ab.

Anhand der Datenbank einer großen kommerziellen US-Krankenversicherung identifizierten die Wissenschaftler vom Brigham and Women's Hospital in Boston 112528 RA-Patienten, welche zwischen 2005 und 2017 mindestens 2 RA-Diagnosen sowie mindestens eine DMARD (disease modifying drug)-Verordnung erhalten hatten. Die Kontrollkollektive bildeten 12837084 Personen aus der Allgemeinbevölkerung, welche nicht an einer RA litten, $2452911 \mathrm{Hy}$ pertoniepatienten, 379900 Arthrosepatienten sowie 15055 Patienten mit einer Psoriasisarthritis. Jede Vergleichsperson hatte ebenfalls mindestens 2 erkrankungsspezifische Diagnosen sowie mindestens eine Verordnung erkrankungsspezifischer Medikamente erhalten. Als primären Studienendpunkt definierten die Forscher die Inzidenz des Typ 2-Diabetes (neue Diabetesdiagnose plus mindestens eine Antidiabetika-Verordnung). Je 108568 Personen der RA-, der Allgemeinbevölkerungs-, der Hypertonie- und der Arthrosegruppe ordneten die Forscher einander bezüglich des Alters, des Geschlechts sowie des Indexdatums zu. In der Psoriasisarthritisgruppe war ein solches Matching mangels ausreichender Fallzahlen nicht möglich.

\section{Ergebnisse}

Das Durchschnittsalter der 108568 RA-, Allgemeinbevölkerungs-, Hypertonie- und Arthrosepatienten betrug 55,6 Jahre und das der Psoriasisarthritispatienten 48,6 Jahre.
Nach einer medianen Nachbeobachtungszeit von 1,6 Jahren stellten die Wissenschaftler die niedrigste Typ 2-Diabetes-Inzidenzrate im Kollektiv der RA-Patienten (7,0 pro 1000 Personenjahre) und die höchste im Kollektiv der Hypertoniepatienten (12,3 pro 1000 Personenjahre) fest. In der Allgemeinbevölkerung, im Arthrosekollektiv und im Psoriasisarthritis-Kollektiv betrugen die Inzidenzraten 7,4, 7,8 bzw. 9,9 pro 1000 Personenjahre. Nach Adjustierung bezüglich mehr als 40 Basis-Kovariablen (z. B. demografische Parameter, Komorbiditäten, Medikamentenanwendung, Inanspruchnahme von Gesundheitsleistungen) errechnete sich für das RA-Kollektiv im Vergleich zur Allgemeinbevölkerung ein signifikant geringeres Typ 2-Diabetesrisiko (Hazard Ratio 0,72; $95 \%$ KI 0,66-0,78). Ähnliches galt für den Vergleich mit der Hypertoniekohorte (Hazard Ratio 0,65; $95 \%$ KI 0,60-0,71), der Arthrosekohorte (Hazard Ratio 0,75; $95 \% \mathrm{KI}$ 0,69-0,81) sowie der Psoriasisarthritis-Kohorte (Hazard Ratio 0,76; 95 \% KI 0,67-0,86). Dies entsprach einer Risikoabnahme im Vergleich zu den 4 Kontrollkollektiven zwischen 24 und $35 \%$.

\section{FAZIT}

Die Ergebnisse der großen populationsbasierten Studie belegen, so das Fazit der Autoren: Patienten mit einer rheumatoiden Arthritis weisen sowohl im Vergleich zur Allgemeinbevölkerung als auch zu Patienten mit einer Hypertonie, einer Arthose oder einer Psoriasisarthritis ein deutlich geringeres Risiko für einen Typ 2-Diabetes auf. Zukünftige Studien müssen nun ihrer Ansicht nach klären, inwiefern sich eine behandelte und eine nicht behandelte RA bezüglich des Diabetesrisikos unterscheiden.

Dr. med. Judith Lorenz, Künzell 